\title{
Dichlorvos Exposure Aggravates Complications Associated with Diabetes in Wistar Rats
}

\author{
${ }^{1}$ Adebayo L. Adedeji, ${ }^{1}$ Ayodele Ogunniyi, ${ }^{2}$ Dorcas Wusu, ${ }^{1}$ Olusegun K. Afolabi ${ }^{*}$ \\ ${ }^{1}$ Department of Biochemistry, Ladoke Akintola University of Technology, Ogbomoso, Nigeria \\ ${ }^{2}$ Lagos State University, Lagos, Nigeria \\ *okafolabi@lautech.edu.ng
}

\begin{abstract}
The high incidence of diabetes and widespread use of the pesticide, dichlorvos (DDVP) make their co-occurrence plausible in the general population. This study was therefore, undertaken to investigate effects of dichlorvos exposure on lipid homeostasis, oxidative status and hepato-renal functions in streptozotocin-induced diabetic rats. DDVP was orally administered at $8 \mathrm{mg} / \mathrm{kg} \mathrm{bw}\left(1 / 10^{\text {th }} \mathrm{LD}_{50}\right)$ to both normal and diabetic rats. Exposure to the organophosphate caused an aggravation of the hypertriglycridemic outcome in diabetic rats while also increasing their phospholipid contents. Induced diabetes lowered HDL-C concentration, while increasing the LDL and VLDL contents. DDVP enhanced concentration of the triglyderide-rich lipoprotein, $V L D L$ in the diabetic animals. DDVP exposure and STZ-induced diabetes reduced HMG CoA reductase activity, however, their co-occurrence brought about a stimulation of the enzyme. Lipid hydroperoxides level was highest in DDVP-exposed diabetic rats while plasma total antioxidant capacity was drastically reduced. Moreover, DDVP escalated production of advanced oxidized protein products in diabetic rats. Pronounced nephrotoxic effects were observed in dichlorvos-exposeddiabetic rats, evidenced by increased blood urea and creatinine. Similarly, activities of plasma transaminases, AST and ALT, markers of hepatic damage were markedly elevated.The findings of this study imply that DDVP has the tendency to exacerbate complications associated with diabetes.
\end{abstract}

Keywords: Dichlorvos, diabetes, dyslipidemia, hepatotoxicity, oxidative stress, nephrotoxicity

Abbreviations: Dichlorvos (DDVP), streptozotocin (STZ), triglyceride (TG), High density lipoprotein (HDL), low density lipoprotein ( $L D L)$, very low density lipoprotein (VLDL), organophosphorous (OP), aspartate aminotransferase (AST), alanine aminotransferase (ALT), ferric reducing/antioxidant power (FRAP), ferric reducing/antioxidant power (FRAP), advanced oxidized protein products (AOPP), hydroxymethylglutaryl CoA (HMG CoA) .

\section{INTRODUCTION}

Pesticides are chemical substances used to control pests in agriculture and households [1]. Among the common class of pesticides are the organophosphorous (OP) compounds. Their extensive use in agriculture and public health has resulted in large scale environmental pollution [2]. Their uncontrolled use in many developing and some developed countries has led to serious health issues among the population [3, 4]. The potential adverse impact of pesticides on human health may be higher in developing countries due to easy accessibility to these chemicals and reduced risk awareness among the populace. In addition to their application in crop protection, organophosphates are widely used to control parasitic vectors of both humans and animals [5]. As a result of their wide application, there is a growing public concern about the accumulation of these insecticides in food products and water supply [6]. Exposure to OP pesticides is known to produce adverse effects in the affected populations. Such toxicological effects include acute cholinergic syndromes, cardiac conduction disorders and delayed neurotoxicity [7]. Several studies have also demonstrated the possible effect of chronic exposure on reproductive function and on neuropsychiatric function [8,9]. Recent studies have observed hyperglycemia as an effect due to OP exposure in both experimental animals and humans $[10,11]$.

Diabetes is a major and growing health concern globally and hyperglycemia, along with dyslipidemia, is a major factor contributing to the development of micro- and macrovascular complications of the disease [12]. Although the etiology of diabetes is not well defined, recent evidences indicate that environmental toxicants may play a role in its induction. Apart from studies showing OPs ability to 
disrupt glucose homeostasis, which can lead to hyperglycemia [11], these class of chemicals have been demonstrated to alter lipid homeostasis in animals [13]. These suggest that OPs, apart from playing a part in diabetes induction, may play an interactive role in the manifestations of secondary complications in diabetes.

Among the most frequently used organophosphates is dichlorvos (2,2-dichlorovinyl dimethyl phosphate; DDVP) which exerts its toxicity by inhibiting acetyl cholinesterase activity and accumulate acetylcholine in the synaptic junction [14].Besides being a neurotoxicant, DDVP causes lipid peroxidation by the generation of free radicals through the production of reactive species and through its metabolism [15], leading ultimately to oxidative stress. Exposure to DDVP can occur through air, water or food and it is rapidly absorbed via all routes of exposures [16].

The prevalence of diabetes in the general population makes it plausible that a significant number of such individuals are exposed to the pesticide, DDVP owing to its widespread use. There are however, no information available on dichlorvos exposure in diabetic condition. Earlier studies have demonstrated that diabetes modifies or alters the pharmacodynamics and pharmacokinetics of drugs or xenobiotics in man and animals $[17,18]$. This study therefore, set to investigate the influence of the interaction between diabetes and dichlorvos exposure on lipid metabolism, hepatorenal functions and degree of oxidative damage. Plasma lipid profile, hepatic and renal damage, as well as, levels of lipid hydroperoxidation, oxidative proteins and total antioxidant capacity, were assessed in normal and streptozotocin-induced diabetic rats exposed to repeated oral doses of DDVP.

\section{MATerials AND Methods}

\subsection{Chemicals}

The organophosphorous compound, dichlorvos was obtained from Saro Agrosciences Limited, Nigeria. Streptozotocin was procured from Sigma-Aldrich Chemical Co. (Germany). All other chemicals used were of analytical grade.

\subsection{Animal Treatment}

Experimental protocols were conducted in accord with guidelines of the Institutional Animal Care and Use Committee and were approved by the Animal Ethical Committee of the Department of Biochemistry, LAUTECH, Nigeria.

Twenty four male adult Wistar rats weighing $150 \pm 10 \mathrm{~g}$ were used for the study. They were housed in an animal room with normal controlled temperature $\left(22 \pm 2{ }^{\circ} \mathrm{C}\right)$ and a regular $12 \mathrm{~h} \mathrm{light}$-dark cycle (06:00-18:00 h). They were allowed 14 days to acclimatize before the commencement of the experiment. The animals were maintained on a standard pellet diet.

\subsection{Experimental Procedure}

The rats were divided into 4 groups of 6 animals each. The first group served as Control and received corn oil at a dose of $1 \mathrm{ml} / \mathrm{kg}$ body weight (bw) via oral gavage. The second group were administered DDVP only at $8 \mathrm{mg} / \mathrm{kg}$ bw/day $\left(1 / 10 \mathrm{LD}_{50}\right.$ oral dose) each day in corn oil and given via oral gavage for 30 days. The third group were administered a single dose of streptozotocin (STZ, $60 \mathrm{mg} / \mathrm{kg} \mathrm{bw}$ i.p) dissolved in $10 \mathrm{mM}$ citrate buffer [19] to induce diabetes. Animals in the fourth group (DDVP+STZ) were treated just like the STZ group and after the onset of diabetes, were administered DDVP as in group two.

At the end of the treatment, animals were sacrificed by cervical dislocation. Blood was collected by cardiac puncture into heparinized tubes and centrifuged to separate the plasma. The liver and kidney were excised and homogenized (10\%, w/v) in $0.15 \mathrm{M}$ Tris- $\mathrm{HCl}$ buffer $(\mathrm{pH} 7.4)$ and centrifuged (3000g for $10 \mathrm{~min}$ ).

\section{BIOCHEMICAL ANALYSES}

\subsection{Assessment of Plasma Lipid Profile}

Cholesterol, triglyceride, phospholipids, and HDL-C in plasma were determined using standard assay kits (CHEMELEX, Spain). The lipoproteins LDL and VLDL were determined using Friedwald formular [20]. LDL-C and VLDL-C fractions were calculated as VLDL-C $=\mathrm{TG} / 5$ and LDLC $=\mathrm{TC}-$ (HDL-C + VLDL-C), respectively.

\subsection{Determination of Hepatic HMG-CoA Reductase Activity}

This was determined according to the method of Rao and Ramakrishnan [21] by measuring the hepatic concentration of HMG-CoA and mevalonate.The ratio of HMG-CoA to mevalonate is taken 
as an index of the activity of HMG-CoA reductase. An increase in this ratio indicates inhibition of cholesterogenesis while a decrease indicates enhanced cholesterogenesis.

\subsection{Assessment of Hepatic Damage}

The extent of hepatic damage was evaluated by measuringplasma activities of aspartate aminotransferase (AST) and alanine aminotransferase (ALT) according to the method described by Reitmann and Frankel [22].

\subsection{Assessment of Renal Damage}

The levels of plasma urea and creatinine were estimated according to the method of Jendrassik and Grof [23].

\subsection{Assessment of Oxidative Damage}

Total antioxidant capacity in plasma was determined using the ferric reducing/antioxidant power (FRAP) assay [24]. Spectrophotometric determination of plasma advanced oxidized protein products (AOPP) levels was performed according to the method by Watko et al. [25]. Concentrations of lipid hydroperoxides (LOOH) in plasma were assessed using the method of Naurooz-Zadeh et al. [26].

\subsection{Statistical Analysis}

Mean and standard deviation (SD) were determined for all parameters and the results were expressed as mean $\pm \mathrm{SD}$. The data were analyzed using analysis of variance (ANOVA) followed by Tukey's HSD test for comparison of means to determine the significance of differences among the groups. $p$ values below 0.05 were considered as significantly different.

\section{RESUltS}

Total cholesterol, triglyceride and phospholipids levels in plasma are represented in Figure $\mathbf{1 .}$ Cholesterol levels were significantly $(\mathrm{p}<0.05)$ increased in both STZ group and DDVP+STZ group (34\% and 35\%, respectively) compared to that of the control. Exposure to DDVP alone caused a 17\% reduction in cholesterol concentration but seemed not to influence the lipid's level in DDVP+STZ rats. Triglyceride was markedly raised in all the treated groups compared to the control. Diabetes induced a significant increase in plasma triglyceride content, elevating it from $55.34 \pm 2.76 \mathrm{mg} / \mathrm{dl}$ in the control to $107.54 \pm 2.03 \mathrm{mg} / \mathrm{dl}$ in STZ group. This represent an increase of $94 \%$ over the control, compared to DDVP group with an increase of $20 \%$. On the other hand, the DDVP exposure in diabetic rats resulted in a profound $191 \%$ increase in totalcholesterol levels in the animals (Fig. 1). Plasma phospholipids concentrations were altered in all the groups, except in DDVP exposure, when compared with the control. The increases were $41 \%$ and 55\% in STZ-induced diabetic group and DDVP+STZ group, respectively.

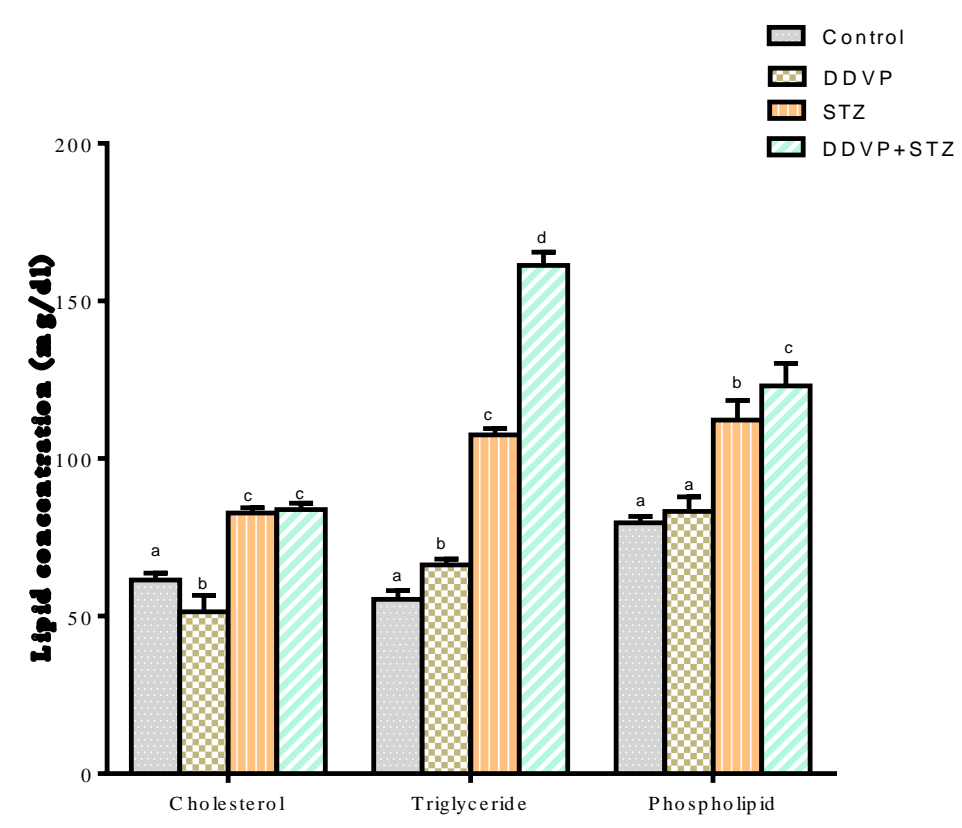

Fig1. Plasma concentrations of cholesterol, triglyceride and phospholipids in Control, DDVP-exposed, STZinduced diabetic and DDVP+STZ rats. Each bar represents mean $\pm S D$ of 6 rats. Bars in the same group bearing different alphabets differ significantly from each other $(p<0.05)$ 
The lipoprotein contents were all significantly altered in all the treatment groups (Fig. 2). DDVP treatment resulted in a slight but significant depressionin HDL-C levels in the rats $(24.12 \pm 0.59 \mathrm{mg} \mathrm{dl}$ and $21.62 \pm 0.63 \mathrm{mg} / \mathrm{dl}$, respectively). The cholesterol content of HDL was reduced by $45 \%$ in STZinduced diabetic group, with the cholesterol content of HDL decreasing to $13.42 \pm 1.09 \mathrm{mg} / \mathrm{dl}$, while the DDVP+STZ group was not significantly different from the STZ group (Fig. 2). With the exception of the DDVP group, LDL-C levels significantly $(p<0.05)$ increased in the treatment groups. DDVP exposure reduced the LDL-C in the rats by $37 \%$ but STZ-induced diabetes increased LDL-C concentration from $26.32 \pm 2.93 \mathrm{mg} / \mathrm{dl}$ to $47.09 \pm 1.12 \mathrm{mg} / \mathrm{dl}$, an increase of $79 \%$. DDVP exposure in STZ-induced diabetes yielded an increase of $51 \%$.Conversely, the DDVP+STZ group was observed to have the highest level of VLDL, which was more than $190 \%$ than the control. This was followed by the diabetic group (STZ) with nearly a 2-fold increase in VLDL over the control and finally, the DDVP group having a significant $(\mathrm{p}<0.05) 20 \%$ more than the control $($ Fig. 2).

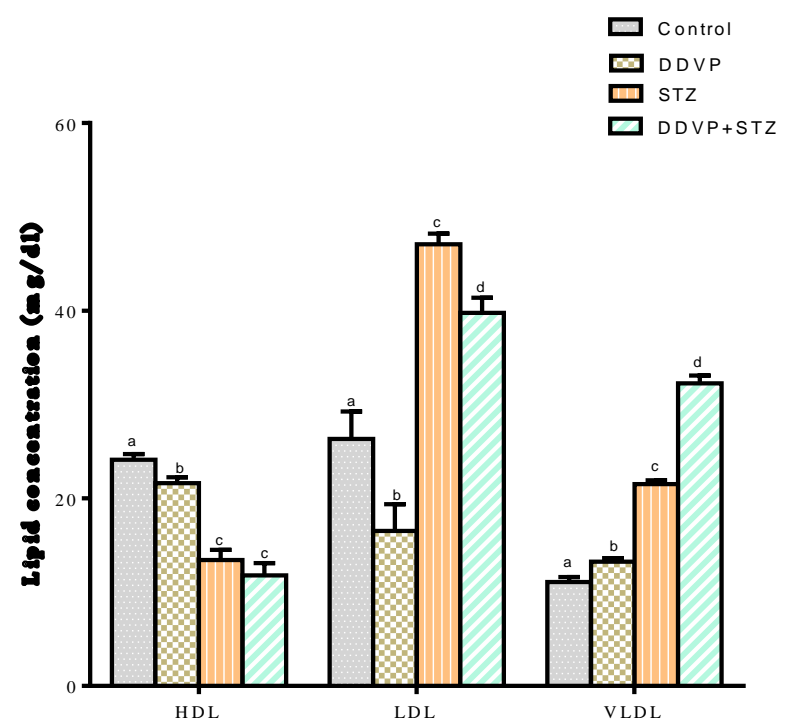

Fig2. HDL, LDL and VLDL concentrations in Control, DDVP-exposed, STZ-induced diabetic and DDVP+STZ rats. Each bar represents mean $\pm S D$ of 6 rats. Bars in the same group bearing different alphabets differ significantly from each other $(p<0.05)$

The HMG CoA reductase activity in livers of the rats is depicted in Fig. 3. Significant increase in the ratio of HMG CoA to mevalonate was observed in both DDVP and STZ groups $(\mathrm{p}<0.05)$. But the ratio was drastically reduced on exposure of DDVP to the diabetic rats (DDVP+STZ group) compared with the control group.

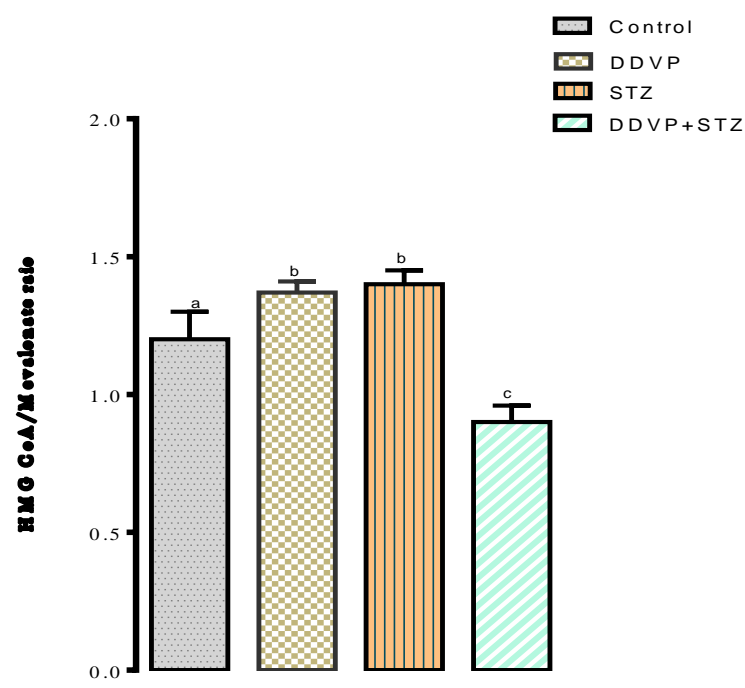

Fig3. HMG CoA reductase activity in Control, DDVP-exposed, STZ-induced diabetic and DDVP+STZ rats. Each bar represents mean $\pm S D$ of 6 rats. Bars in the same group bearing different alphabets differ significantly from each other $(p<0.05)$.

*Lower ratio indicates higher enzyme activity and higher values indicate lower enzyme activity. 
Induction of diabetes resulted in significant elevation of plasma levels of the hepatic transaminases (Fig. 4). STZ resulted in 37\% and 15\% increase in both ALT and AST activities, respectively. DDVP yielded a slight increase in ALT but more significant elevation of the AST activity. On the other hand, exposure to DDVP to diabetic rats significantly increased the levels of both ALT and AST to $98 \%$ and $64 \%$, respectively.

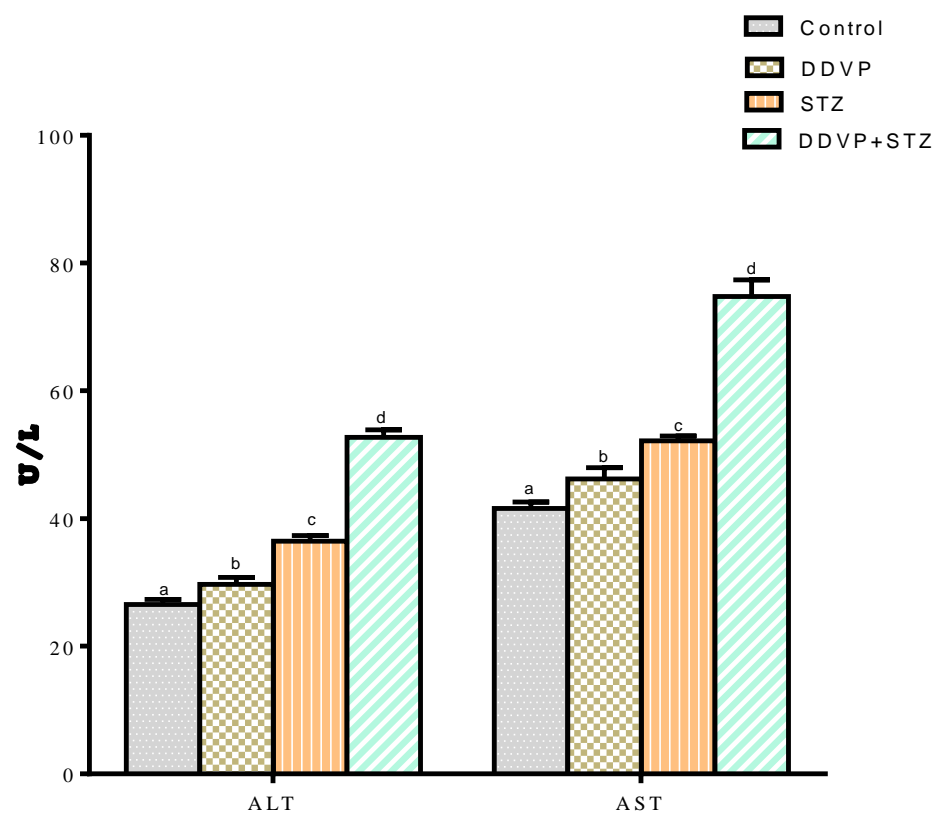

Fig4. ALT and AST activities in Control, DDVP-exposed, STZ-induced diabetic and DDVP+STZ rats. Each bar represents mean $\pm S D$ of 6 rats. Bars in the same group bearing different alphabets differ significantly from each other $(p<0.05)$

Table 1 shows that DDVP treatment, as well as, STZ-induced diabetes caused abnormal kidney function as reflected in the elevation of both BUN an creatinine in the plasma of the rats. DDVP exposure in diabetic state resulted in significantly $(p<0.05)$ higher concentrations of these metabolites in the rats.

Table1. Blood urea nitrogen (BUN) and creatinine in Control, DDVP-exposed, STZ-induced diabetic and $D D V P+S T Z$ rats

\begin{tabular}{|l|l|l|}
\hline Group & BUN $(\mathbf{m g} / \mathbf{d l})$ & Plasma creatinine $(\mathbf{m g} / \mathbf{d l})$ \\
\hline Control & $38.33 \pm 5.79^{\mathrm{a}}$ & $0.59 \pm 0.05^{\mathrm{a}}$ \\
\hline DDVP & $47.59 \pm 2.48^{\mathrm{b}}$ & $0.71 \pm 0.05^{\mathrm{b}}$ \\
\hline STZ & $64.13 \pm 1.79^{\mathrm{c}}$ & $0.95 \pm 0.06^{\mathrm{c}}$ \\
\hline DDVP + STZ & $103.30 \pm 3.63^{\mathrm{d}}$ & $1.48 \pm 0.10^{\mathrm{d}}$ \\
\hline
\end{tabular}

Values are mean $\pm S D ; n=6$. Values not bearing matching superscript on the same row are significantly different from each other at $p<0.05$.

The plasma antioxidant power, oxidized protein contents and lipid peroxidation levels are shown in Table 2. The antioxidant power measured as FRAP, was $61 \%, 45 \%$ and $64 \%$ reduced in DDVP, STZ and DDVP+STZ, respectively, while AOPP contents were elevated by $43 \%, 108 \%$ and $238 \%$, respectively. The level of lipid peroxidation was higher in the DDVP+STZ group than either DDVP or STZ group.

Table2. Lipid hydroperoxides ( $L O O H)$, advanced oxidized protein products (AOPP) and ferric reducing antioxidant power (FRAP) in Control, DDVP-exposed, STZ-induced diabetic and DDVP+STZ rats

\begin{tabular}{|l|l|l|l|l|}
\hline Parameters & Control & DDVP & STZ & DDVP + STZ \\
\hline LOOH $(\mu \mathrm{mol} / \mathrm{L})$ & $12.35 \pm 1.55^{\mathrm{a}}$ & $23.22 \pm 1.07^{\mathrm{b}}$ & $23.70 \pm 1.34^{\mathrm{b}}$ & $30.59 \pm 2.01^{\mathrm{c}}$ \\
\hline AOPP $(\mu \mathrm{mol} / \mathrm{L})$ & $167.50 \pm 10.59^{\mathrm{a}}$ & $239.82 \pm 12.05^{\mathrm{b}}$ & $348.88 \pm 23.88^{\mathrm{c}}$ & $565.91 \pm 33.38^{\mathrm{d}}$ \\
\hline FRAP $(\mu \mathrm{mol} / \mathrm{mL})$ & $240.41 \pm 10.72^{\mathrm{a}}$ & $93.39 \pm 5.13^{\mathrm{b}}$ & $132.22 \pm 18.34^{\mathrm{c}}$ & $86.33 \pm 11.74^{\mathrm{d}}$ \\
\hline
\end{tabular}

Values are mean $\pm S D ; n=6$. Values not bearing matching superscript on the same row are significantly different from each other at $p<0.05$. 


\section{DISCUSSION}

The present study showed that dichlorvos exposure and streptozotocin-induced diabetic rats exhibited dyslipidemia, hepatotoxicity, nephrotoxicity and oxidative stress. But these trends were more pronounced as indicated by dysregulation in lipid metabolism, altered hepato-nephrotoxic markers and heightened oxidant products in STZ-induced diabetic rats than DDVP exposed rats. Data from this study also demonstrated the exaggerated effect of DDVP induced toxicity in the diabetic animals.

In this study, DDVP triggered an increase in plasma triglyceride contents in the rats. Organophosphates, including DDVP have already been reported to elevate plasma triglyceride [27, 28]. The elevated plasma triglyceride concentration may be attributed to DDVP induced inhibition of lipoprotein lipase. On the other hand, dyslipidemia is one of the most common complications in diabetes and it plays a significant role in the progression of the disease [29]leading to morbidity and mortality. Such lipid derangement, including hypertriglyceridemia and high concentrations of LDL, were exhibited by the STZ-induced diabetic rats in this study.Interestingly,exposure of the diabetic rats to DDVP markedly increased the plasma triglyceride concentration in an additive manner. This implies that the organophosphate has the potential to exacerbate diabetes-induced dyslipidemia.

Hypercholesterolemia has been associated with organophosphate exposures in a number of studies $[30,31]$. But there has been contradictory reports about the effect of some of these organophosphates on plasma cholesterol levels. In agreement with the reports of Lucic et al. [32]and Ryhanen et al. [27], our study demonstrated that DDVP lowered plasma cholesterol concentration in rats. This could be attributed to the inhibition of hydroxymethylglutaryl CoA reductase, the rate limiting enzyme in cholesterol biosynthesis, by the organophosphate, DDVP through phosphorylation [27]. This was collaborated by the reduced activity of HMG CoA reductase, reflected as an increase in the ratio of HMG CoA to mevalonate, observed in DDVP-exposed rats. In addition, DDVP could also have stimulated the LDL receptors thereby, increasing the rate of cholesterol clearance from circulation [33]. On the other, hypercholesterolemia which is linked with a greater risk for the development of atherosclerosis and its attendant complications, is the hallmark of advanced diabetic condition. STZinduced diabetic rats were observed with increased plasma cholesterol contents in this study, but with a reduction in HMG CoA activity. The increase in plasma cholesterol concentration in diabetic rats could have resulted from a reduction in the clearance rate of the lipid. It is of interest, however, that exposure of these diabetic rats to DDVP did not alter or affect the induced hypercholesterolemic state in the animals. The high cholesterol level in plasma is suggestive of liver damage and has been associated with the development of coronary artery disease and other organ complications [34]. Defect in secretion of bile juice into the duodenum may occur in such damaged liver, thus preventing the catabolism of cholesterol and giving raise to the high level of the lipid in the blood. Of significance is the increase in HMG CoA reductase activity seen in this DDVP+STZ group, which could explain the hypercholesterolemia in the animals. The co-occurrence of DDVP and diabetes seemed to have resulted in a stimulation of the enzyme in a yet unexplained mechanism.Phospholipid concentrations were elevated in both STZ and DDVP+STZ groups, with the increases probably induced by diabetes. The action of the lipolytic hormones on the peripheral fat instigated by insulin results in the hydrolysis of triglyceride and prevents the mobilization of free fatty acids. Inactivation of lipoprotein lipase through insulin deficiency promotes the conversion of free fatty acids into phospholipids which is then discharged into the blood, resulting in the elevated plasma phospholipids [35].

Studies have indicated hypercholesterolemia, hypertriglyceridemia, elevated LDL levels, and decreased HDL-C in diabetes [36]. HDL is synthesized mainly in the liver and it facilitates cholesterol efflux from tissues to the liver for removal as bile acids [37]. In contrast toreports on organophosphates where the lipoprotein concentration was increased [38, 27], HDL level was slightly depressed by DDVP exposure in this study. On the other hand, diabetic rats displayed a significant decrease in HDL-C below that of the control, a level that was not changed by DDVP exposure.Taking into account the increased levels of VLDL and phospholipids in the present study, the low concentration of HDL-C in diabetes could be contributed by the increased hepatic secretion of VLDL resulting from rapid free fatty acid flux to the liver [39, 40]. Through the action of cholesterol ester transfer protein, triglyceride could have been transferred from VLDL to HDL, creating triglycerideenriched HDL particles, which on hydrolysis by hepatic lipase and would be rapidly cleared from 
plasma [41]. The increase in LDL-C levels in the diabetic rats is major caused by an increased mobilization of the free fatty acids from peripheral deposits due to the deficiency of insulin. This increase in the plasma LDL-C level may also result from glycosylation of the lysyl residues of apoprotein $\mathrm{B}$, with the attendant decrease in LDL metabolism due to a decrease in the affinity of LDL for its receptors.

Diabetes is marked by increased generation of free radicals or diminished antioxidant defenses. Reactive hydroxyl radicals are formed as a result of the production of superoxide anion radicals by glucose oxidization and its dismutase to hydrogen peroxide [42, 43]. Extensive data have also shown that organophosphates produce oxidative stress and the oxygen free-radical formation can be a major contributor to the toxicity of these pesticides [44, 45]. Oxidative damage occurs through the production of reactive oxygen species such as $\mathrm{H}_{2} \mathrm{O}_{2}$, hydroxyl radicals, and superoxide anion. These eventually disturb the equilibrium status of pro-oxidant/antioxidant status resulting in oxidative stress in the cellular system. In this study, total antioxidant capacity measured as FRAP was significantly reduced in DDVP exposure than that in the diabetic rats. Conversely, the level of plasma AOPPs was greater in diabetic rats than in DDVP exposure, while lipid hydroperoxides concentrations were not significantly different between the two groups. However, DDVP exposure in diabetic state effected the most pronounced toxicity responses. A significant decrease in the total antioxidant level and a concomitant increase in $\mathrm{LOOH}$ level with the elevation of advanced protein products in co-occurrence of DDVP poisoning and diabetes, indicate a heightened state of oxidative stress in this group. This could have been precipitated by glucose oxidation and increased formation of free radicals in the diabetic rats, in tandem with the depletion of the antioxidant system during DDVP detoxification, which has been suggested as a mechanism by which DDVP increases oxidative stress [46]. Consequently, the attenuation of antioxidant defenses could result in the amassing of reactive oxygen species and other free radicals, increasing lipid peroxidation and protein oxidation as evidence by the elevated levels of $\mathrm{LOOH}$ and AOPP in the rats.

Reactive oxygen species (ROS) have been implicated in DDVP toxicity potentially leading to liver dysfunction [47]. Liver is an organ of metabolism and detoxification and it is known to be a target for toxic agents. Organophosphates have been reported to induce hepatotoxicity [48] and the hepatic dysfunction is accompanied by elevated levels of plasma marker enzymes indicating cellular leakage and loss of functional integrity of hepatic membrane [49]. This correlates with our study, which showed increased activities of AST and ALT in the plasma of DDVP exposed rats. STZ-induced diabetes also resulted in an elevation of both hepatic markers in the rats, but at a rate higher than that observed in the DDVP-treated rats. The diabetic-DDVP exposed rats however, displayed a much higher degree of hepatic damage than either groups, indicating that their co-occurrence could aggravate damage to the liver. This clearly denotes a tendency of DDVP to exacerbate hepatic damage in diabetic state.

Reactive oxygen species and oxidative damage to biological macromolecules are known to also play important role in development of nephrotoxicity [50]. Studies have also reported higher level of Nacetyl-B-glucosamine (NAG), an early indication of kidney dysfunction in diabetes subjects [51]. Nephrotoxicity was evaluated by estimating BUN level, a marker of proximal tubule injury and creatinine level, a marker of glomerular injury [52]. Studies have demonstrated organophosphate induced nephrotoxicity in humans and experimental animals [53].The increase in blood urea and creatinine of both DDVP and STZ-induced diabetic rats in the present study is in agreement with the report of Hadi et al.[54] and Patel and Kalia [52]. Increased catabolism of both liver and kidney proteins may account for the diabetes associated elevated BUN [55]. While earlier studies have demonstrated the propensity of organophosphates to induce nephrotoxicity in humans occupationally exposed to these compounds [56]. From our data, the degree of nephrotoxicity was greater in diabetic rats than in DDVP exposed rats, as evident from higher level of BUN and creatinine. However, DDVP exposure to diabetic rats led to a more pronounced nephrotoxic response in the animals, showing that DDVP aggravates renal damage in diabetic rats.

\section{Conclusion}

Overall, these findings depict DDVP with having the potential to aggravate dyslipidemia, exhibited through hypertriglyceridemia and increased triglyceride-enriched lipoproteins in diabetic state. Also, the organophosphate magnifies diabetes-associated hepatorenal toxicity and oxidative stress, suggesting that DDVP may increase the risk of developing renal and hepatic complications in diabetic 
sub-population susceptible to exposure to this organophosphate. Further studies are however, required to ascertain the mechanisms by which DDVP provoked these responses in diabetic condition.

\section{REFERENCES}

[1] B.C. Gbaruko, E.I. Ogwo, J.C. Igwe, H. Yu, Organophosphate induced chronic neurotoxicity: health, environmental and risk exposure issues in developing nations of the world, Afr. J. Biotechnol. 8 (2009) 5137-5141.

[2] A.M. Abu El-Saad, M.M Ibrahim, A.A. Hazani, G.A. El-Gaaly, Lycopene attenuates dichlorvosinduced oxidative damage and hepatotoxicity in rats, Human Exp. Toxicol. 35(6) (2015) 654-65.

[3] S. Singh, N. Sharma, Neurological syndromes following organophosphate poisoning, Neurol. India. 48 (2000) 308-313.

[4] M. Lotti, A. Moretto, Organophosphate-induced delayed polyneuropathy, Toxicol. Rev. 24 (2005) 37-49.

[5] N. Dwivedi, G. Flora, P. Kushwaha, S.J.S. Flora, Alpha-lipoic acid protects oxidative stress, changesin cholinergic system and tissue histopathologyduring co-exposure to arsenic-dichlorvos in rats, Environ Toxicol Pharmacol. 37 (2013) 7-23.

[6] S. Ince, I. Kucukkurt, H.H. Demirel, R. Turkmen, E. Sever, Thymoquinone attenuates cypermethrin induced oxidative stress in Swiss albino mice, Pestic Biochem Physiol. 104(2012) 229-235.

[7] E. Michael, A.B. Nick, E. Peter, H.D. Andrew, Mangement of acute organophosphorous poisoning, Lancet 371(2008) 597-607.

[8] A. Okamura, M. Kamijima, E. Shibata, K. Ohtani, K. Ueyama, Y. Watanbe, M. Omura, H. Wang, G. Ichihara, T. Kondu, T. Nakajima, A comprehensive evaluation of the testicular toxicity of dichlorvos in Wistar rats, Toxicology 213 (2005) 129-137.

[9] R.M. Salvi, D.R. Lara, E.S. Ghisolfi, L.V. Portela, R.D. Dias, D.O. Souza, Neuropsychiatric evaluation in subjects chronically exposed to organophosphate pesticides, Toxicol. Sci. 72 (2003) 267-271.

[10] J. Seifert, Toxicological significance of the hyperglycemia caused by organophosphorous insecticides, Bull. Environ. Contam. Toxicol. 67 (2001) 463-469.

[11] R. Rahimi, M. Abdollahi, A review on the mechanisms involved in hyperglycemia induced by organophosphorus pesticides, Pest. Biochem. Physiol. 88 (2007) 115-121.

[12] R. Rahimi,S. Nikfer, B. Larijani, M. Abdollahi, A review on the role of antioxidants in the management of diabetes and its complications, Biomed. Pharmacol. 59 (2008) 365-373.

[13] M.M. Lasram, A.B. Annabi, N.E. Elj, S. Selmi, A. Kamoun, S.L. Fazza, N. Gharbi, Metabolic disorders of acute exposure to malathion to adult Wistar rats, J. Hazard Mater. 163 (2009) 10521055 .

[14] B.K. Binukumar, K.D. Gill, Cellular and molecular mechanisms of dichlorvos neurotoxicity: cholinergic, noncholinergic, cell signaling, gene expression and therapeutic aspects, Indian J. Exp. Biol. 48 (2010) 697-709.

[15] N. Dwivedi, S.J.S. Flora, Concomitant exposure to arsenic and organophosphates on tissue oxidative stress in rats, Food Chem. Toxicol. 49 (2011) 1152-1159.

[16] G. Raheja, K.D. Gill KD, Calcium homeostasis and dichlorvos induced neurotoxicity in rat brain, Mol Cell Biochem. 232 (2002) 13-18.

[17] K.E. Thummel, J.B. Schenkman, Effects of testosterone and growth hormone treatment on hepatic microsomal P450 expression in the diabetic rat, Mol Pharmacol. 37 (1990) 119-129.

[18] K. Chawalit, P. Stret Argusa, A. Thitapanda, Comparative effects of diabetogenic agents on hepatic drug metabolism, Drug. Metab. Dispos. 10 (1982) 81-86.

[19] N. Singh, V. Kamath, P.S. Rajini, Attenuation of hyperglycemia and associated biochemical parameters in STZ-induced rats by dietary supplementation of potato peel powder, Clin. Chim. Acta. 353 (2005) 165-175.

[20] W.T. Friedwald, R.J. Levy, D.S. Fredricken, Estimation of VLDL-cholesterol in the plasma without the use of preparative ultracentrifuge, Clin. Chem. 18(1972) 449-502. 
[21] A.V. Rao, S. Ramakrishnan, Indirect assessment of hydroxymethylglutaryl- CoA reductase (NADPH) activity in liver tissue, Clin Chem.21(1975)1523-1525.

[22] S. Reitman, S. Frankel, A colorimetric method for determination of serum glutamic-oxaloacitic and glutamicpyruvic transaminase, Am. J. Clin. Pathol.28 (1957) 56-68.

[23] L.P. Jendrassik,P. Grof, Vereinfachte photometrische methoden zur bestimmung des blutbilirubins. Biochemische Zeitschrift Band. 297 (1983) 81-89.

[24] I.F.F. Benzie, J.J. Strain, The ferric reducing ability of plasma (FRAP) as a measure of antioxidant power: The FRAP assay. Anal. Biochem. 239 (1996) 70-76.

[25] V. Witko-Sarsat, M. Friedlander, C. Capeillere-Blandin, T. Nguyen-Khoa, A.T. Nguyen, J. Zingraff, P. Jungers, B. Descamps-Latscha, Advanced oxidation protein products as a novel marker of oxidative stress in uremia, Kidney International. 49 (1999) 1304-1313.

[26] J. Nourooz-Zadeh, J. Tajaddini-Sarmadi, S.P. Wolff, Measurement of plasma hydroperoxide concentrations by the ferrous oxidation-xylenol orange assay in conjunction with triphenylphosphine, Anal Biochem. 220 (1994) 403-409.

[27] R. Ryhanen, J. Herranen, K. Karhonen,I. Penttila,M. Popvilanpi,E. Puhakainen, Relationship between serum lipids, lipoproteins and pseudocholinesterase during organophosphate poisoning in rabbits, Int. J. Biochem. 16 (1984) 687- 690.

[28] N.A. Ibrahim, B.A. El-Gamal, Effects of diazinon, an organophosphate insecticide, on plasma lipid consitutents in experimental animals, J. Biochem. Mol. Biol. 36 (2003) 499-504.

[29] I.J. Goldberg, N.A. Le, J.R. Paterniti, H.N. Ginsberg, F.T. Lindgren, W.V. Brown, Lipoprotein metabolism during acute inhibition of hepatic triglyceride lipase in the Cynomolgus monkey. J. Clin. Invest. 70 (1982) 1184-1192.

[30] P.D. Choudhary, C.H. Chakraharti, Effects of acephate (Orthene), an organophosphorus insecticide on lipid metabolism in albino rats, Indian J. Exp. Biol. 22 (1984) 359-367.

[31] M.A. Yehia, S.G. El-Banna, A.B. Okab, Diazinon toxicity affects histophysiological and biochemical parameters in rabbits, Exp. Toxicol. Pathol. 59 (2007) 215-225.

[32] A. Lucić, V. Bradamante, B. Radić, M. Peraica, A.M. Domijan, R. Fuchs, A. StavljenićRukavina, The effect of dichlorvos treatment on butyrylcholinesterase activity and lipid metabolism in rats, Arh Hig Rada Toksikol. 53(2002) 275-281.

[33] M.S. Brown, P.T. Kovanen, J.L. Goidstein, Regulation of plasma cholesterol by lipoprotein receptors, Science. 212 (1981) 628-635.

[34] H.M. Abdou,R.H. El-Mazoudy, The Myotoxic and Hyperlipidemic Effect of Diazinon in Female Rats. J. Medical Res. Inter. 28 (2007) 292-298.

[35] P.N. Pushparaj, H.K. Low, J. Manikandan, B.H.H. Tan, C.H. Tan, Anti-diabetic effects of Cichorium intybus in streptozotocin-induced diabetic rats, J. Ethnopharmacol, 111(2007) 430434.

[36] B.V. Howard, D.C. Robbins, M.L. Sievers, E.T. Lee, D. Rhoades, R.B. Devereux, L.D. Cowan, R.S. Gray, T.K. Welty, O.T. Go, W.J. Howard,LDL cholesterol as a strong predictor of coronary heart disease in diabetic individuals with insulin resistance and low LDL: the Strong Heart Study,Arterioscler. Thromb. Vasc. Biol. 20(2000) 830-835.

[37] A.R. Shakoori, S.S. Ali, M.A. Saleem, Effects of Six Months Feeding of Cypermethrin on the Blood and Liver of Albino Rats, J. Biochem. Toxicol. 3 (1988) 59-72.

[38] C. Uchendu, S.F. Ambali, J.O. Ayo, I.O. Lasisi,A.J. Umosen, Subacute chlorpyrifos-induced alterations in serum lipids and some oxidative stress biomarkers in male Wistar rats: beneficial effect of acetyl-L-carnitine, Toxicol. Environ. Chem. 95(2013) 483-494.

[39] R.M. Krauss, P.W. Siri, Dyslipidemia in type 2 diabetes. Med. Clin. N. Am. 88 (2004)897-909.

[40] A. Laws, H.M. Hoen, J.V. Selby, M.F. Saad, S.M. Haffner, B.V. Howard, Differences in insulin suppression of free fatty acid levels by gender and glucose tolerance status: relation to plasma triglyceride and apolipoproteins B concentrations: Insulin Resistance Atherosclerosis Study (IRAS) Investigators, Arterioscler. Thromb. Vasc. Biol. 17 (1997) 64-71.

[41] G.J. Hopkin, P.J. Barter, Role of triglyceride- rich lipoproteins and hepatic lipase in determining the particle size and composition of high density lipoproteins, J Lipid Res. 27 (1986) 1265-1277. 
[42] Z.Y. Jiang, A.C.S. Woollard, S.P. Wolff, Hydrogen peroxide production during experimental protein glycation, FEBS Letters. 268(1990) 69-71.

[43] S.P. Wolff,R.T. Dean, Glucose autoxidation and protein modification. The potential role of autoxidative glycosylation in diabetes, Biochem. J. 245(1987) 243-250.

[44] D. Bagchi, J. Balmoori, M. Bagchi, X. Ye, C.B. Williams, S.J. Stohs, Comparative effects of TCDD, endrin, naphthaleneand chromium (IV) on oxidative stress and tissue damage in the liver and brain tissues of mice, Toxicology 175 (2002) 73-82.

[45] T.O. Ajiboye, Redox status of the liver and kidney of 2,2-dichlorovinyl dimethyl phosphate (DDVP) treated rats. Chem. Biol. Interact. 185 (2010) 202-207.

[46] T.M. Bui-Nguyen, C.E. Baer, J.A. Lewis, D. Yang, P.J. Lein,D.A. Jackson, Dichlorvos exposure results in large scale disruption of energy metabolism in the liver of the zebrafish, Danio rerio. BMC Genomics. 16 (2015) 853.

[47] B.K. Binukumar, A. Bal, R. Kandimalla, A. Sunkaria, K.D. Gill, Mitochondrial energy metabolism impairment and liver dysfunction following chronic exposure to dichlorvos, Toxicology. 270 (2010) 77-84.

[48] A. Ogutcu, Z. Suludere, Y. Kalender, Dichlorvos induced hepatotoxicity in rats and the protective effects of vitamins C and E, Environ. Toxicol. Pharm. 26 (2008) 355-361.

[49] L. Pari,A. Prasath, Efficacy of caffeic acid in preventing nickel induced oxidative damage in liver of rats. Chem. Biol. Interact. 173 (2008) 77-83.

[50] M. SinhA, P. Manna, P.C. Sil, Arjunolic acid attenuates arsenic-induced nephrotoxicity, Pathophysiology. 15 (2008) 147.

[51] J.P. Wang, S.L. Wang, Q. Lin, L. Zhang, D. Huang, J.C Ng, Association of arsenic and kidney dysfunction in people with diabetes and validation of its effects in rats, Environ. Int. 35(2009) 307.

[52] H.V. Patel,K. Kalia, Sub-chroic arsenic exposure aggravates nephrotoxicity in experimental diabetic rats, India J. Exp. Biol. 48 (2010) 762-768.

[53] M.A. Attia, Risk assessment of occupational exposure to pesticides, Earth Environ. Sci. 3 (2006) 349-362.

[54] M.A. Hadi, E.H. Hameedi, N.J. Kadhum, D.A. Aziz, A.H. Al-Saddi, H.K. Zaidan, Ameliorative Effect of Curcuma longa L. Rhizomes against biochemical toxicity induced by dichlorvos in female albino aats. J. Chem. Pharm. Sci. 9(2016) 1098-1106.

[55] P.K. Dabla, Renal function in diabetic nephropathy, World J. Diabetes. 15 (2010) 48-56.

[56] A. Jorda, M. Gomez, J. Carbo,S. Grisolia, Effects of streptozotocin diabetes on some urea cycle enzymes, Biochem. Biophys. Res. Commun. 106 (1982) 37-43. 